\title{
ARTIGO ORIGINAL \\ Vinculação da gestante com a maternidade: a influência no tipo de parto
}

Elisandra de Cássia Popolli*, Jessica Mayara Barcellos*, Juliana Rodrigues Zuco*, Tatiana Aparecida Ribeiro Coelho*, Luciana Braz de Oliveira Paes, M.Sc. ${ }^{* *}$, Paulo Fasanelli***, Zaida Aurora Sperli Geraldes Soler, D.Sc. ${ }^{\star * * \star}$

\begin{abstract}
*Egressa do Curso de Enfermagem do Centro Universitário Padre Albino (UNIFIPA), ${ }^{* *}$ Enfermeira Obstétrica, Docente do Centro Universitário Padre Albino (UNIFIPA), Catanduva/SP, ${ }^{* \star *}$ Médico obstetra, mestrando do Programa de Pós-Graduação Stricto Sensu em Enfermagem, Mestrado Acadêmico da Faculdade de Medicina de São José do Rio Preto/SP (FAMERP), ${ }^{\star * \star * O b s t e t r i z, ~ e n f e r m e i r a, ~ l i v r e-d o c e n t e ~ e m ~ e n f e r m a g e m ~ o b s t e ́ t r i c a, ~}$ docente e orientadora de graduação e pós-graduação lato sensu e stricto sensu da FAMERP
\end{abstract}

Recebido em 18 de junho de 2018; aceito em 30 de junho de 2018.

Endereço para correspondência: Luciana Braz de Oliveira Paes, Rua Barão do Rio Branco, 436 Centro 15828000 Palmares Paulista SP, E-mail: lucianabrazenf@gmail.com; Elisandra de Cássia Popolli: elisandrapopoli@gmail.com; Jessica Mayara Barcellos: jmbascellos30@mail.com; Juliana Rodrigues Zuco: juzucco@gmail.com; Tatiana Aparecida Ribeiro Coelho: tatycoelho.ribeiro@gmail.com; Paulo Fasanelli: paulofasanelli@hotmail.com; Zaida Aurora Sperli Geraldes Soler: zaidaaurora@gmail.com

\section{Resumo}

Introdução: A vinculação da gestante com a maternidade é tapropriada para o nascimento humanizado e contribui para aumento dos partos normais, sendo um direito garantido por lei no Brasil. Objetivo: Verificar a opinião de gestantes sobre a vinculação da gestante com a maternidade e a influência quanto ao parto de eleição. Métodos: Pesquisa de caráter exploratório, descritiva e análise de dados quali-quantitativa. Os sujeitos do estudo foram gestantes que visitaram a maternidade campo de pesquisa, localizada em um município de médio porte do interior paulista. Resultados: Entrevistadas 31, destacando-se os dados: faixa etária de 21-30 anos (41,9\%); escolaridade de 12 ou mais anos (45,2\%); desempregadas $(61,29 \%)$; todas faziam acompanhamento pré-natal e consideravam importante conhecer a maternidade para o parto; multíparas (54,8 \%); 93,5\% escolhiam o parto vaginal e nos relatos de valorização da visita à maternidade ressaltaram acolhimento, segurança e receber orientações sobre o parto. Conclusão: A vinculação da gestante com a maternidade influênciou na escolha do tipo de parto, com preferência pelo parto normal.

Palavras-chave: humanização do parto, parto normal, maternidade, obstetrica.

\begin{abstract}
Women's link with maternity services and its influence on mode of delivery

Introduction: The creation of a link between maternity services and pregnant women contributes to the humanization of childbirth and the increase of normal delivery. Moreover, getting to know a maternity facility prior to delivery is a right secured by law. Objective: To investigate women's opinion on how the link with a maternity facility influences their choice of delivery. Methods: This is an exploratory, descriptive study and qualitative-quantitative data analysis. The subjects of the study were pregnant women who visited the maternity research field, located in a mediumsized municipality in the interior of São Paulo. Results: Thirty-one women were interviewed. Of these, $41.9 \%$ were aged $21-30$ years; $45.2 \%$ had completed 12 or more years of schooling; $61.29 \%$ were unemployed; $54.8 \%$ were multiparae; $93.5 \%$ chose vaginal delivery as the preferred mode of delivery. All of the women were in prenatal care and believed it was important to get to know the maternity facility before onset of labor. Participants valued this prenatal visit because it helped them feel safe and welcomed, and provided them with guidance on childbirth. Conclusion: A woman's link with the maternity facility influences her delivery mode decisions and favors natural delivery.
\end{abstract}

Key-words: humanization of childbirth, normal birth, maternity, obstetrics. 


\section{Resumen \\ La vinculación de la gestante con la maternidad y su influencia en el tipo de parto elegido}

Introducción: Además de ser un derecho reconocido por ley, la creación de un vínculo entre la gestante y la maternidad contribuye a la humanización del parto y al aumento de partos normales. Objetivo: Conocer la opinión de las gestantes acerca de la vinculación con la maternidad y su influencia en el tipo de parto elegido. Métodos: Investigación de carácter exploratorio, descriptivo y análisis de datos cual-cuantitativos. Los sujetos del estudio fueron gestantes que visitaron la maternidad campo de investigación, ubicada en un municipio de mediano porte del interior paulista. Resultados: Se entrevistaron 31 embarazadas. El 41,9\% tenían entre 20 y 30 años de edad; el 45,2\% tenían 12 o más años de escolaridad; el $61,29 \%$ estaban desempleadas; el 54,8\% eran multíparas; y el 93,5\% elegían el parto vaginal. Todas las entrevistadas recebían cuidado prenatal y consideraban importante conocer la maternidad antes del parto. Según las mujeres, tal visita les hace sentir seguras y acogidas, y les permite obtener orientación sobre el parto. Conclusión: La vinculación de la gestante con la maternidad influye en la elección del tipo de parto y favorece el parto normal.

Palabras-clave: humanización del parto, parto normal, maternidad, obstetricia.

\section{Introdução}

A cada ano acontecem no Brasil cerca de três milhões de nascimentos, 98\% em ambiente hospitalar, o que significa que ao menos seis milhões de binômios - mãe e filhovivenciam mais diretamente este momento, via de regra não prazeroso [1,2].

Ao longo das últimas décadas, pelo menos a partir de 1990, o Brasil é mencionado em todo o mundo como o país das cesáreas e de prática obstétrica intervencionista, hospitalocêntrica, medicalizada, que permitiu a concretização de um modelo que considera a gravidez, o parto e o nascimento como doença e não como expressões de saúde [3,4].

No atual cenário do parto, houve ganhos no acesso a tecnologias de saúde que impactaram de forma positiva na saúde da mulher e da criança, porém algumas intervenções, que deveriam ser utilizadas com critério, são rotineiras e atingem quase a totalidade das mulheres e seus filhos assistidos nos hospitais no Brasil. Assim, são desconsiderados os aspectos emocionais, humanos e culturais envolvidos neste processo, com repercussões negativas na saúde física e emocional da mulher, da criança e da família [5].

A institucionalização do parto culminou com a desorganização da assistência obstétrica, que hoje envolve necessariamente o deslocamento da gestante em busca de resolução do parto, percorrendo uma rede de saúde complexa e na maioria das vezes desarticulada. Ainda, transformou e interferiu nas relações do cuidado à saúde e nas interações interpessoais mulher-bebê-família, deslocando o protagonismo para a equipe de saúde no lugar da mulher e da família [6].

Em 27 de dezembro de 2007 foi sancionada a Lei oㅜ 11.634, que dispõe sobre o direito da gestante ao conhecimento e a vinculação à maternidade, assim como receber assistência no âmbito do Sistema Único de Saúde (SUS). Em seu artigo 1ํ destaca que toda gestante tem direito ao conhecimento e à vinculação prévia à maternidade na qual será realizado seu parto será atendida nos casos de intercorrências no pré-natal e também no puerpério [7].

Então, mesmo "desconhecida"em muitos hospitais e por muitos profissionais, a vinculação da gestante à maternidade é um direito garantido por lei no Brasil. Entretanto, o direito assegurado por lei e a sua transformação em exercício constituinte incorporado na rotina da vida apresenta dificuldades para serem superadas, sendo esta uma das propostas do Ministério da Saúde, formalmente, desde 2000, integrando o Programa de Humanização do Parto e Nascimento do Ministério da Saúde[8].

Além da humanização no nascimento, a vinculação é uma tecnologia apropriada para o parto, pois deve interferir no processo fisiológico do trabalho de parto, ao dirimir ou diminuir o medo, a insegurança, a desinformação, as incertezas, as dúvidas, a falta de apoio. Vale lembrar que situações de estresse interferem na liberação de hormônios importantes do processo do parto, como a ocitocina endógena, importante para o trabalho de parto eutócico, enquanto em situações de estresse há maior liberação de adrenalina, hormônio do medo, dificultando a evolução normal do parto. De modo geral, a vinculação da gestante à maternidade é uma tecnologia apropriada ao nascimento, um recurso que influencia a prática obstétrica humanizada, já que estimula a fisiologia do trabalho de parto e propicia mais 
segurança, confiança e tranquilidade para o adequado transcorrer do parto e resolução do parto normal [6].

A forma de nascer não significa apenas a via de parto, deve ser entendida como foco de atenção fundamental para o futuro e o desenvolvimento das relações humanas. Envolve toda a ambiência relacionada ao processo do nascimento, desde o domicílio, a atenção primária de saúde, o hospital/maternidade, a vinculação com os serviços e as equipes, o acolhimento com deslocamento seguro para o parto [9].

$\mathrm{Na}$ busca de agir contra a desumanização da prática obstétrica instalada no Brasil, várias iniciativas foram sendo realizadas, entre outras [10,11]: em junho de 2011 o Governo Brasileiro instituiu a Rede Cegonha no âmbito do SUS (Sistema Único de Saúde), visando assegurar à mulher o direito ao planejamento reprodutivo e à atenção humanizada à gravidez, ao parto e ao puerpério. E adotou a adoção de práticas de atenção à saúde baseada em evidências científicas nos termos do documento da Organização Mundial da Saúde, de 1996: "Boas práticas de atenção ao parto e ao nascimento" [10,11].

Com as "Boas Práticas do Parto e Nascimento", aprovada pela Resolução SS - 42, de 06-05-2015, que assegura o direito ao parto humanizado, torna-se de extrema importância à adoção de diretrizes que resgatem à mulher o protagonismo do parto e nascimento, baseado no respeito aos seus direitos, assegurando-lhe privacidade e apoio emocional, oferecido também por seu familiar de escolha durante todo o processo de parturição [12].

Sendo assim, entende-se que todas as instituições hospitalares sob gestão do Estado, devam adequar para que sejam garantidos os direitos da gestante e atendendo às recomendações da OMS, providenciando: Implantação das Boas Práticas de atenção ao parto e puerpério propostas pela OMS, sendo uma das recomendações "Vincular a gestante à maternidade por meio de regulação no território e dos fluxos de visita à maternidade" [13].

Cabe ressaltar que as visitas à maternidade permitem a redução da ansiedade e desmistificação do parto normal e do aleitamento materno, além da participação do acompanhante durante o parto, o conhecimento prévio das normas institucionais, o estímulo à cidadania das usuárias e a vinculação da usuária à maternidade são considerados medidas importantes da humanização [14]. Ante o exposto, este estudo tem como objetivo verificar a importância da vinculação da gestante com a maternidade e sua opinião quanto ao tipo de parto desejado e influência das orientações quanto ao tipo de parto.

Material e métodos

Trata-se de pesquisa de caráter exploratório, descritiva e com apresentação e análise de dados quali-quantitativa. Os dados foram registrados em um formulário semi-estruturado, elaborado pelas autoras da pesquisa e aplicado em forma de entrevista. Os sujeitos do estudo foram gestantes que visitaram a maternidade campo de pesquisa, por ocasião da visita a maternidade definida para atendimento ao parto e intercorrências na gestação e puerpério, localizada em um município de médio porte do interior paulista.

Vale explicar que na instituição hospitalar campo desta pesquisa, as visitas à maternidade são programadas mensalmente e inclui orientação quanto ao tipo de parto, com ênfase no incentivo ao parto normal.

Os resultados quantitativos deste estudo foram apresentados em forma de tabela. Para a análise dos dados qualitativos, as entrevistas foram transcritas na íntegra e submetidas à análise. No tratamento dos resultados, tais elementos foram agrupados, de acordo com a semelhança, para a formação de três categorias temáticas: Orientação recebida quanto ao parto, Segurança e acolhimento, Falta de conhecimento prévio quanto o nascimento.

Esta pesquisa respeitou todas as recomendações de pesquisas envolvendo seres humanos, previstas na Resolução № 466/2012 do Conselho Nacional de Saúde e foi aprovada por Comitê Permanente de Ética em Pesquisa Envolvendo Seres Humanos sob Parecer no69601217.9.0000.5430. Além disso, cada uma das participantes, ao aceitar fazer parte da pesquisa, assinou o Termo de Consentimento Livre e Esclarecido (TCLE).

Resultados e discussão

Os resultados a seguir mostram a relação de algumas variáveis de caracterização amostral de gestantes que visitaram a maternidade. Foram entrevistadas 31 gestantes que consentiram em participar do estudo, destacando-se entre as gestantes do estudo o que se mostra na Tabela I. 
- idade que variou de 15 a 40 anos, com predomínio na faixa de 21 a 30 anos $(41,9 \%)$. Tais dados se assemelham a outro estudo no Brasil, que relata que em nosso meio, desde 2000, uma redução do número absoluto de nascimentos entre mulheres menores de 24 anos e um aumento da idade média das mães, sendo de 25,7 anos em 2007; em 2009, a proporção de nascidos vivos de acordo com a idade da mãe foi de $20 \%$ em menores de 20 anos, $54,4 \%$ em mães entre 20 e 29 anos e $26,7 \%$ em mães com mais de 30 anos [15];

- quanto ao grau de escolaridade, $14(45,16 \%)$ tinham 12 ou mais anos de estudo formal, o que condiz com outra pesquisa, que evidenciou que o aumento da escolaridade materna tem acompanhado o aumento da escolaridade da população feminina. No Brasil, 8,2\% das mães dos recém-nascidos em 2009 tinham entre zero e três anos de escolaridade, $28,7 \%$ tinham entre quatro e sete anos e $63,1 \%$ tinham oito anos ou mais de escolaridade [16];

- a respeito da ocupação/profissão, a maioria se classificou como desempregada $(61,3 \%)$, enquanto $06(19,4 \%)$ tinham trabalho formal remunerado. O trabalho da mulher em todo o mundo está continuamente se transformando, em decorrência das mudanças relacionadas à maternidade, à estrutura familiar, ao casamento e ao divórcio, da urbanização, dos avanços tecnológicos e da globalização da economia [17];

- sobre a procedência, $24(77,42 \%)$ gestantes residiam em cidades da região, e sete $(22,6 \%)$ no município estudado. Esse resultado é intrigante, mas explica-se, pois municípios menores viabilizam o transporte das mulheres às maternidades de referência para o parto, e muitas vezes são acompanhadas por enfermeiras ou outros profissionais da área da saúde. Aliás, o enfermeiro é profissional estratégico para cumprir com as diretrizes para atenção pré-natal estabelecida pelo Programa de Humanização no Pré-Natal e Nascimento (PHPN), e Rede Cegonha [18].

Tabela I - Percentuais referentes aos dados sociodemográficos das gestantes que visitaram a maternidade. Catanduva, São Paulo, 2017.

\begin{tabular}{|c|c|c|}
\hline \multicolumn{3}{|l|}{ Faixa etária } \\
\hline 10 a 14 anos & 00 & 0,00 \\
\hline 15 a 20 anos & 11 & 35,48 \\
\hline 21 a 30 anos & 13 & 41,94 \\
\hline 31 a 40 anos & 07 & 22,58 \\
\hline 41 a 50 anos & 00 & 0,00 \\
\hline \multicolumn{3}{|l|}{ Escolaridade } \\
\hline Nenhuma & 00 & 0,00 \\
\hline 1 a 4 anos & 02 & 6,45 \\
\hline 5 a 8 anos & 06 & 19,35 \\
\hline 9 a 11 anos & 09 & 29,03 \\
\hline$\geq 12$ anos & 14 & 45,16 \\
\hline \multicolumn{3}{|l|}{ Ocupação } \\
\hline Trabalho doméstico próprio & 03 & 9,68 \\
\hline Trabalho informal & 00 & 0,00 \\
\hline Trabalho formal & 06 & 19,35 \\
\hline Desempregada & 19 & 61,29 \\
\hline Outros & 03 & 9,68 \\
\hline \multicolumn{3}{|l|}{ Procedência } \\
\hline Cidade do estudo & 07 & 22,58 \\
\hline Cidades da região & 24 & 77,42 \\
\hline
\end{tabular}

Verifica-se na Tabela II:

- $96,8 \%$ das gestantes tinham até 22 semanas de gestação, no segundo trimestre. A procura da mulher pela assistência durante o ciclo gravídico-puerperal tem aumentado no Brasil, observando-se aumento da cobertura no pré-natal a partir dos anos 2000, o que é um indicador importante de atenção à saúde materna e infantil [15]. Pode-se dizer que a cobertura da assistência pré-natal no Brasil é praticamente universal, com valores elevados em todas as regiões do país e em mulheres de diferentes características demográficas, sociais e reprodutivas. Por outro lado, nem remotamente isso significa ou reflete melhoria na qualidade dessa atenção [18]; 
- $45,2 \%$ eram primigestas e $38,7 \%$ tiveram de um a quatro partos, mostrando que tanto primíparas quanto multíparas buscam informações durante a gestação. Em outra pesquisa foi destacado que mulheres multíparas apresentaram risco duas vezes maior por não buscarem acesso às informações e consultas de pré-natal, comparadas às primíparas [19];

- sobre as intercorrências na gestação atual, $24(77,4 \%)$ afirmaram que não tiveram problemas na gestação atual, enquanto os agravos relatados pelas outras foram: hipertensão arterial, hipertensão arterial e diabetes gestacional e hipertensão arterial e anemia, sífilis e toxoplasmose. Refletindo, a adesão das mulheres ao serviço de saúde independentemente da característica materna, pois a realização da assistência prénatal no Brasil foi de $98,7 \%$, sendo superior a $90 \%$ em todos os grupos, seja de alto risco ou de risco habitual [19].

Tabela II - Percentuais referentes aos dados obstétricos das gestantes que visitaram a maternidade. Catanduva, São Paulo, 2017.

\begin{tabular}{lcc}
\hline Idade Gestacional & 30 & \\
Até 22 semanas & 01 & 96,77 \\
23 a 27 semanas & 00 & 0,00 \\
28 a 31 semanas & 00 & 0,00 \\
32 a 36 semanas & 00 & 0,00 \\
37 a 41 semanas & 00 & 0,00 \\
42 semanas & & \\
Gestação & 14 & 45,16 \\
01 & 15 & 48,39 \\
02 a 04 & 02 & 6,45 \\
05 a 08 & & 58,06 \\
Paridade & 18 & 38,71 \\
00 & 12 & 3,32 \\
01 a 04 & 01 & \\
05 a 08 & & 80,65 \\
Aborto & 25 & 19,35 \\
00 & 06 & 0,00 \\
01 a 04 & 00 & 100 \\
05 a 08 & & 0,00 \\
Acompanhamento pré-natal & 31 & \\
Sim & 00 & 77,42 \\
Não & & 3,23 \\
Intercorrência gestacional & & 3,23 \\
Nenhuma & 24 & 3,23 \\
Hipertensão Arterial & 01 & 6,45 \\
HA + Diabete Gestacional & 01 & 3,23 \\
HA + Anemia & 01 & 3,23 \\
Sangramento vaginal & 02 & \\
Sífilis & 01 & \\
Toxoplasmose & 01 & \\
\hline
\end{tabular}

Todas as gestantes destacaram a importância da visita à maternidade onde serão atendidas no parto, emergindo falas apresentadas em três categorias: Orientações quanto ao parto, Segurança e acolhimento, Conhecimento prévio quanto o nascimento.

\section{Orientações recebidas quanto ao parto}

Revela o déficit do conhecimento sobre o parto, a falta de orientação quanto ao parto normal e deficiência de informações nas consultas de pré-natal.

\footnotetext{
“... Foi importante conhecer e tirar dúvida sobre o parto...”.

“... tirei várias dúvidas sobre o parto e descobri que não é nada daquilo que as pessoas falam...".

"... Sim, porque explicou como é o parto que agora tem coisas para relaxar...".

"... Sim, tirei as dúvidas sobre o parto e como que vai acontecer...".
} 


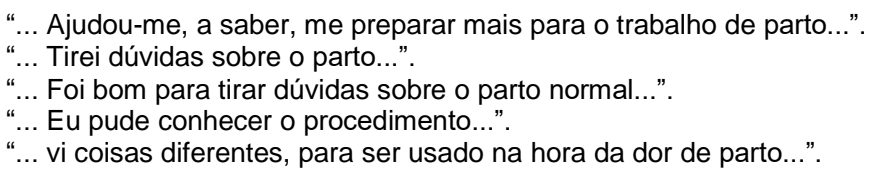

A gestante deve receber orientações e informações sobre a gravidez, o parto e 0 puerpério no decorrer das consultas de pré-natal quanto ao tipo de parto, desde os aspectos técnicos, referentes ao trabalho corporal, incluindo rotinas e procedimentos da maternidade referência, até aspectos cognitivos e emocionais. Para isso, os profissionais envolvidos nos serviços de pré-natal devem adotar medidas educativas [18,20]. Há necessidade de considerar na qualidade de atenção pré-natal não só o número de consultas, mas também as orientações fornecidas para a redução dos índices de morbimortalidade materna e perinatal [21].

As gestantes neste estudo manifestaram preferência pelo parto normal após visitarem a maternidade, após receberem informações sobre métodos de alívio da dor baseados nas "Boas práticas de atenção ao parto". Das informações que as gestantes recebem no pré-natal pouco se fala sobre práticas para facilitar o parto, que são de relevância para a promoção do parto vaginal, sendo priorizadas durante o pré-natal as orientações sobre sinais de risco, reforçando o caráter biomédico da assistência [18].

\section{Segurança e valorizar o acolhimento}

Mencionada tanto no sentido do serviço oferecer o nascimento seguro, quanto a mulher sentir-se segura principalmente quando cita a importância do acompanhante.

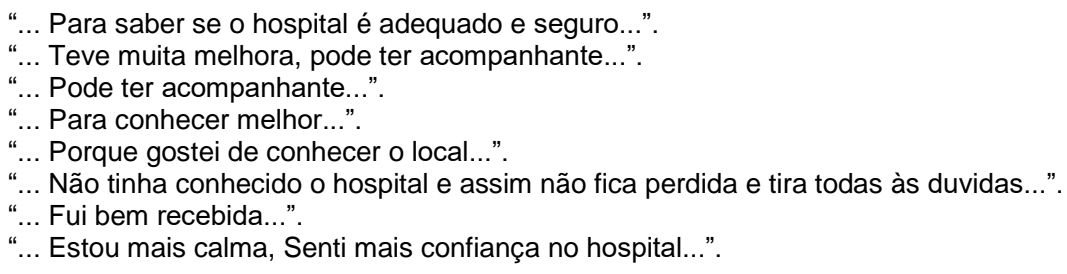

A equipe obstétrica precisa priorizar a humanização durante $o$ atendimento a gestante, entendendo-a como prática pautada em princípios como integralidade e equidade das ações, evidenciando os usuários como sujeitos de direitos e participantes ativos do seu processo saúde/doença. Quando o atendimento é feito de forma contextualizada e qualificada, além de se basear nos aspectos clínicos e científicos, proporciona também o acolhimento da mulher e família, valorizando e respeitando a individualidade de cada uma, considerando as múltiplas dimensões que circundam o viver em sociedade, a criação de vínculos e a participação ativa das mulheres no momento do pré- natal, parto e puerpério [21].

Emergiu nas falas das gestantes a importância do acompanhante, destacado em muitas pesquisas. A gestação tem sido associada a um período de ansiedade para mulher e a família devido ao período de transição diante do nascimento e o acompanhante no momento do parto é de muita ajuda. Além do mais, esse é um direito garantido legalmente no Brasil, - Lei no11. 108, promulgada em 7 de abril de 2005, que assegura a presença do acompanhante de escolha da mulher durante o processo de nascimento. Não se pode negar ao pai ou acompanhante de eleição da mulher o direito de vivenciar um dos momentos mais importantes da vida do ser humano, o nascimento de um novo membro da família [22].

\section{A falta de conhecimento prévio quanto ao nascimento}

No que tange à ótica da gestante, devido à falta de conhecimento prévio quanto ao nascimento, ocorreram manifestações sobre modificações do período perinatal e a estrutura física da maternidade, o que influência no bem-estar deste período.

\footnotetext{
“... Não conhecia e tirou muitas dúvidas...".

“... Desenvolvi conhecimentos...”.

"... Pelos conhecimentos gerais...".

“... Para poder conhecer coisas que eu não sabia...”.

".. Para a gente saber melhor...”.

"... Aprendi muitas coisas que não sabia...".
} 
“... Foi importante conhecer a maternidade para mim e meu bebê...".

As alterações fisiológicas ocorridas durante a gravidez sejam elas sutis ou marcantes estão entre as mais acentuadas que o corpo humano pode sofrer, gerando medos, dúvidas, angústias, ou curiosidade. É relevante que esses sentimentos sejam compartilhados nas consultas de pré-natal, não se restringindo apenas aos aspectos biológicos da gestação [23].

$\mathrm{Na}$ Tabela III estão dados sobre a preferência das gestantes do estudo, antes e após a visita à maternidade, quanto à via de resolução do parto. Observa-se que $22(71 \%)$ já preferiam o parto vaginal. Após a visita, apenas duas gestantes ainda preferiam a cesariana. Durante a gravidez e o parto as mulheres vivenciam várias alterações físicas e emocionais e expressam neste processo, valores e crenças, assim como se defrontam com a estrutura social e cultural dos serviços de saúde que podem apresentar potencial influência na escolha do tipo de parto [24]. A expectativa das mulheres a respeito da escolha do tipo de parto tem relação com o conhecimento das mesmas sobre $o$ assunto e as informações que são tratadas pelos profissionais da área da saúde. Por isso é importante a troca de conhecimentos duração a realização do pré-natal, possibilitando o esclarecimento de dúvidas, reduzindo a ansiedade em relação às vias de parto, propiciando atenção obstétrica integral e de melhor qualidade [25].

Pudemos analisar neste estudo que as mulheres durante a gestação preferem o parto vaginal quando são informados dos benefícios do mesmo, porém o que acontece no Brasil é que a cesariana é influenciada pela assistência recebida no pré-natal, pela fonte de pagamento do parto e outros fatores socioeconômicos, sugerindo que no Brasil, o parto cirúrgico é um bem de consumo [26].

Como foi percebido também neste estudo, há falta de consonância entre as informações referentes ao parto durante o pré-natal, com a recebida na maternidade. Percebese que existem grandes lacunas da assistência à saúde no Brasil e ausência de uma filosofia de trabalho em equipe, que permita resultados na melhoria dos indicadores de saúde, particularmente na assistência perinatal. Vários resultados adversos de morbimortalidade materna e fetal têm sua origem em um trabalho em equipe fraco e desarticulado [27].

No Brasil, dados da literatura mostram que ao longo das consultas de pré-natal, principalmente no âmbito de atenção privada, os médicos criam um cenário incentivando as mulheres à intervenção na resolução do parto. Destacam nas consultas de pré-natal, riscos mais supostos do que reais como: bebê grande, bacia estreita, circular de cordão, pouco líquido, apresentações anômalas, o que intimidam as gestantes e sua família que "optam" pela cesárea diante do temor de serem responsabilizadas por qualquer desfecho negativo e fragilizadas diante do "poder de convencimento" dos médicos [27].

Muitas cesarianas são indesejadas e a mulheres são "persuadidas" ao final da gestação, com apresentação de quadros de riscos que não condizem com as evidências científicas. Com a "banalização" da cirurgia, as mulheres não se surpreendem com sua indicação e aderem à cesárea, renunciando à vontade inicial pelo parto vaginal [26,27].

Tabela III - Percentuais da preferência das gestantes quanto ao tipo de parto antes e após visita à maternidade. Catanduva, São Paulo, 2017.

\begin{tabular}{lcccc}
\hline Vaginal & 22 & 70,17 & 29 & 93,5 \\
Cesárea & 09 & 29,3 & 02 & 6,5 \\
\hline
\end{tabular}

Conclusão

Segundo o objetivo definido e os resultados obtidos, concluímos neste estudo que a vinculação da gestante com a maternidade influenciou na decisão das gestantes quanto à preferência pelo parto normal. Também, sobre a importância da visita à maternidade, no foco da humanização, pois propicia conhecimento, conforto e segurança para a gestante.

Os resultados desta pesquisa indicam a urgência em reformar o modelo de atenção ao parto e o nascimento, tendo em vista que as orientações quanto ao parto vaginal no pré-natal são falhas.

Vale destacar que o tema da vinculação à maternidade não tem sido pesquisado no Brasil e que o momento atual de atendimento obstétrico na região estudada é muito crítico. Assim, é preciso cumprir de um lado as normas legais vigentes e, de outro, que os profissionais atendam com proficiência técnico-científica, mas também com humanidade, dignidade e respeito, propiciando que as mulheres sejam protagonistas em seu parto. 
1. Brasil. Ms/Svs/Dasis. Nascidos vivos Brasil. Nascim p/resid. Mãe segundo Região. Sistema de Informações sobre Nascidos Vivos - SINASC, 2013. 2016 Jan [citado 2016 Jan 20]. Disponível em:http://tabnet.datasus.gov.br/cgi/tabcgi.exe?Sinasc/cnv/nvuf.deff.

2. Brasil. Ministério da Saúde. Secretaria de Ciência, Tecnologia e Insumos Estratégicos. Departamento de Ciência e Tecnologia. PNDS 2006 - Pesquisa Nacional de Demografia e Saúde da Criança e da Mulher. Brasília: Ministério da Saúde; 2008, p.583.

3. Brasil. MS. Secretaria de ciência, Tecnologia e Insumos Estratégicos. Comissão Nacional de Incorporação de Tecnologias no SUS. Diretrizes de atenção à gestante: a operação cesariana, 2016. [citado 2016 Jan 20]. Disponível em: http://conitec.gov.br.

4. World Health Organization. Trends in maternal mortality: 1990 to 2010 Who, Unicef, Unfpa and The World Bank estimates. Geneva: World HealthOrganization; 2012. p. 59.

5. Goldenberg RL, Mcclure EM. Maternal Mortality. Am J Obstetr Gynecol 2011;293-5.

6. Brasil. Ministério da Saúde. Humanização do parto e do nascimento. Ministério da Saúde. Universidade Estadual do Ceará. Brasília: Ministério da Saúde; 2014. p.465.

7. Brasil. Lei No 11.634, de 27 de Dezembro de 2007. Dispõe sobre o direito da gestante ao conhecimento e a vinculação à maternidade onde receberá assistência no âmbito do Sistema Único de Saúde. Diário Oficial da República Federativa do Brasil, 27 de dez de 2007. Seção 1, p.1.

8. Brasil. Ministério da Saúde. Portaria No 569, de 1 de junho de 2000. Dispõe dos recursos humanos, físicos, materiais e técnicos necessários à adequada assistência ao parto. Diário Oficinal da União, 06 de jun de 2000. Seção 1, p.4-5-6.

9. Lansky S. Por um novo modo de nascer no Brasil, 2010. [citado 2016 Mar 24]. Disponível em: http://portal saude. gov.br/portal/saude/visualizar_texto.cfm?idtxt=35995.

10. Brasil. Ministério da Saúde. Portaria No 1.459, de 24 de junho de 2011. Institui no âmbito do Sistema Único de Saúde - SUS - a Rede Cegonha. Diário Oficial da União, 27 de jun 2011.Seção 1, p.109.

11. Organização Mundial da Saúde. Assistência ao Parto Normal: um guia prático. Genebra: OMS; 2000. p.93.

12. Brasil. Ministério da Saúde. Portaria No 11, de 7 de janeiro de 2015. Redefine as diretrizes para implantação e habilitação de Centro de Parto Normal (CPN), no âmbito do Sistema Único de Saúde (SUS) para o atendimento à mulher a ao recém-nascido no momento do parto e do nascimento, em conformidade com o componente Parto e Nascimento da Rege Cegonha, e dispõe sobre os respectivos incentivos financeiros de investimento, custeio mensal. Diário Oficial da União, 07 de jun de 2015. Seção 1, p.1.

13. Manual de Rede Cegonha. Monitoramento e avaliação de processos, metas com base nas diretrizes e indicadores da rede cegonha. Brasília: Ministério da Saúde; 2015.

14. Neto MC. Pré-natal: aspectos importantes da humanização. Revista Sogesp 2015;120.

15. Brasil. Secretaria de Vigilância em Saúde. Departamento de Análise de Situação em Saúde. Saúde Brasil 2010: uma análise da situação de saúde e de evidências selecionadas de impacto de ações de vigilância em saúde. Brasília: Secretaria de Vigilância em Saúde; 2011.

16. Brasil. Secretaria de Atenção à Saúde. Departamento de Atenção Básica. Atenção ao pré-natal de baixo risco. Brasília: Ministério da Saúde, 2012. Cadernos de Atenção Básica, n. 32.

17. Hilfingermessias DK, Im GO, Page A ,Reger H, Speerl., YoderLI, et al.Defining and redefining work: Implications for women`s health. Gender \& Society [s.d.] p.296-323.

18. Viellas EF. Assistência pré-natal no Brasil. Cad Saúde Pública 2014;30(Sp):S85-S100.

19. Darmont MQR, Martins HS, Calvet GA, Deslandes SF, Menezes JA. Adesão ao prénatal de mulheres HIV+ que não fizeram profilaxia da transmissão vertical: um estudo sócio comportamental e de acesso ao sistema de saúde. Cad Saude Publica 2010;26(9):1p.788-96.

20. Lamy OG, Moreno SB. Assistência Pré-Natal e preparo para o parto. Omnia Saúde 2013;10(2):19-35.

21. Anversa RTE, Bastos NAG, Nunes NL, Dal Pizzol ST. Qualidade do processo da assistência pré-natal: unidades básicas de saúde e unidades de Estratégia Saúde da Família em município no Sul do Brasil. Cad Saúde Pública 2012;(4)789-800. 
22. Souza BV, Roeckerll S, Marcon SS. Ações educativas durante a assistência pré-natal: percepção de gestantes atendidas na rede básica de Maringá-PR. Rev Eletr Enferm 2011;13(2):199-210.

23. Perdomini IRF, Bonilha LLA. A participação do pai como acompanhante da mulher no parto. Texto Contexto Enferm 2015;20(3):445-52.

24. Costa SE, Pinon BMG, Costa ST, Santos ACR, Nóbrega RA, Sousa BL. Alterações fisiológicas na percepção de mulheres durante a gestação. Revista Rene 2010;11(2):86-93.

25. Pires D, Fertonani P H, Matos AT, Cordova PF, Mazur SC. A influência da assistência profissional em saúde na escolha do tipo de parto: um olhar sócio antropológico na saúde suplementar brasileira. Rev Bras Saúde Matern Infant 2010;10(2):191-7.

26. Silva CPS, Prates GCR, Campelo AQB. Parto normal ou cesariana? Fatores que Influenciam na escolha da gestante. Rev Enferm UFSM 2014;4(1):1-9.

27. Domingues RMSM, Dias MAB, Nakamura-Pereira M, Torres JA, d'Orsi E, Pereira APE, et al. Processo de decisão pelo tipo de parto no Brasil: da preferência inicial das mulheres à via de parto final. Cad Saúde Pública 2014;30(Supl.1):S101-16. 